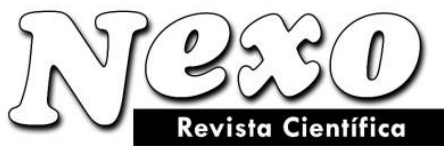

ISSN-E 1995-9516

\title{
Descripción de parámetros fisíco-químicos de la pulpa de café (Coffea arabica) en diferentes variedades $y$ pisos altitudinales
}

\section{Description of physical-chemical parameters of coffee pulp (Coffea arabica) in different varieties and altitudinal floors}

\author{
Jhunior Abrahan Marcia Fuentes ${ }^{1,}{ }^{*}$, Cleiry Estefany Palma Murillo ${ }^{1}$, Mauricio José Torres Loza ${ }^{1}$ \\ Ingris Mary Varela Murillo ${ }^{1}$, Keisy Bianey Peralta Matute ${ }^{1}$, Carlos Humberto Amador Zelaya ${ }^{2}$, \\ Héctor Antonio Díaz Antúnez ${ }^{3}$, Leonardo Antonio Chavarría Carrión ${ }^{4}$ \\ ${ }^{1}$ Universidad Nacional de Agricultura. Facultad de Ciencias Tecnológicas. Catacamas, Honduras. \\ ${ }^{2}$ Universidad Nacional de Agricultura. Facultad de Ciencias Agrarias. Catacamas, Honduras. \\ ${ }^{3}$ Universidad Nacional de Agricultura. Facultad de Medicina Veterinaria. Catacamas, Honduras. \\ ${ }^{4}$ Universidad Nacional de Ingeniería. Facultad de Ingeniería Química. Managua, Nicaragua. \\ *juniorabrahamm@yahoo.com
}

(recibido/received: 01-June-2020; aceptado/accepted: 14-September-2020)

\begin{abstract}
RESUMEN
Este trabajo tuvo como objetivo evaluar los parámetros físicos-químicos y sensoriales de la pulpa de café (Coffea arábica), de las variedades Catuaí, Lempira y Parainema, provenientes de diferentes pisos altitudinales a $1275 \mathrm{msnm}, 1461 \mathrm{msnm}$ y $1550 \mathrm{msnm}$, empleados como parámetros de control de calidad para el desarrollo de bebidas tipo infusión. Se utilizaron muestras homogéneas de $1 \mathrm{~kg}$ de pulpa de cada variedad y piso altitudinal. Las variables de respuesta fueron solidos solubles ( ${ }^{\circ} \mathrm{Brix}$ ), potencial de hidrogeno $(\mathrm{pH})$ y perfil en taza a partir de pruebas descriptivas con jueces expertos de la empresa Café Orgánico Márcala, Sociedad Anónima (COMSA). Los resultados indicaron que la variedad Catuaí presentó la mejor respuesta en la interacción variedad-altitud con aceptación sensorial, determinando valores de $15.9^{\circ} \mathrm{Bx}$, un $\mathrm{pH}$ de 5.45 , en un piso altitudinal de $1550 \mathrm{msnm}$, además reflejo mejor perfil en taza con un $83 \%$ describiendo una tonalidad a frutas. La interacción de estas variables más el control de la cinética de fermentación del grano, condicionan la calidad final de la bebida tipo infusión.
\end{abstract}

Palabras clave: Café; Parámetros físico-químicos; Pisos altitudinales; Perfil en taza; Infusión.

\section{ABSTRACT}

The objective of this work was to evaluate the physical-chemical and sensorial parameters of the coffee pulp (Coffea arabica), of the Catuaí, Lempira and Parainema varieties, coming from different altitude levels at 1275 masl, 1461 masl and 1550 masl, used as parameters of quality control for the development of infusion-type beverages. Homogeneous samples of $1 \mathrm{~kg}$ of pulp of each variety and altitudinal floor were used. The response variables were soluble solids $\left({ }^{\circ}\right.$ Brix $)$, hydrogen potential $(\mathrm{pH})$ and cup profile from descriptive tests with expert judges from the company Organic Coffee Márcala, Anonymous Society (COMSA). The results indicated that the Catuai variety presented the best response in the variety-altitude interaction, determining values of $15.9^{\circ} \mathrm{Bx}$, a $\mathrm{pH}$ of 5.45 , at an altitude level of 1550 masl, also reflecting a better profile in cup with $83 \%$ describing a fruit hue. The interaction of these variables plus the control of the fermentation kinetics of the grain determine the final quality of the infusion type drink.

Key words: Coffee; Physico-chemical parameters; Altitudinal floors; Profile in cup; Infusion. 


\section{INTRODUCCIÓN}

En la actualidad, el café es uno de los principales rubros a nivel agroindustrial de gran importancia, tanto en el mercado nacional como internacional. En los países pocos desarrollos el valor de este preciado grano es relativamente bajo, ya sea por mala comercialización a otros países o por la poca diversificación de productos que se pueden desarrollar a base del café, en Honduras, se registra en los últimos años que el café ha sido uno de los principales productos de exportación, siendo el país con mayor exportación en Centroamérica y uno de los cinco principales países exportadores del mundo (IHCAFÉ, 2017). En la industria del café, solo el $9.5 \%$ del peso de la fruta fresca se utiliza en la preparación de la bebida y el $90.5 \%$ en forma de residuos. La pulpa es la parte más voluminosa del grano de café, representa entre 40 y $56 \%$ en peso, además de contener agua en $20 \%$, cáscara en $4.5 \%$ y mucílago en $16 \%$ (Coronel y Marín, 2010).

Por cada dos toneladas de café se genera alrededor de una tonelada de pulpa, que es rica en nutrientes como azúcares, proteína, fibra y minerales. Sin embargo, la pulpa de manera general es empleada como compostaje, como fuente de energía renovable y ensilaje, sin embargo, este residuo no siempre es destinado para dichas alternativas, por el contrario, es desechado sin control (IHCAFÉ, 2017). La pulpa es el primer bioproducto que se obtiene en el procesamiento de la fruta del café, según datos de la FAO, a nivel mundial los residuos de la industria del café se han estimado en aproximadamente 22 millones de toneladas de pulpa de café y 8.6 millones de toneladas de mucílago, estos bioproductos son descargados en ríos y causan contaminación (Ulloa et al., 2002).

La pulpa de café puede ser considerada como una materia prima con elevado contenido de compuestos de interés, siendo una planta medicinal y su consumo en infusiones puede ayudar a la prevención de enfermedades degenerativas, teniendo en cuenta que se ha establecido una relación entre el consumo de estos polifenoles y la reducción de riesgos de padecer enfermedades crónicas no transmisibles, incluyendo obesidad y diabetes (Mozaffarian, 2016). Serna et al., (2018); declaro valores de 356.78 $\mathrm{mg} / \mathrm{L}$ de fenoles extraídos de la pulpa de café, siendo mayor en comparación con el té negro que cuantifica valores de compuestos fenólicos entre 200 y $350 \mathrm{mg} / \mathrm{L}$.

Según Marcía et al., (2020); las plantas medicinales tienen varias condiciones benéficas para la humanidad, una de ellas es su aporte medicinal debido a la presencia de fitoquímicos y antioxidantes, caracterizados por estos compuestos bíoactivos como la principal fuente de nutracéuticos. Además, el aprovechamiento de la pulpa de café para el desarrollo de bebidas tipo infusión, con características nutracéuticas, es una alternativa económica de alto impacto tecnológico y ambiental, que genera mayor aprovechamiento del fruto (Marcía et al., 2019a).

El cultivo del café crece y se desarrolla dentro de un rango térmico de 18 a $22^{\circ} \mathrm{C}$ debido a las influencias altitudinales (FAO, 1985; Quintero et al., 2016). Según Jaramillo (2005); en Centroamérica en los países productores de café los pisos altitudinales para el cultivo oscilan entre 700 y $1700 \mathrm{~m}$ respectivamente. En Cuba se reportó que, a mayor altitud, precipitación y humedad relativa, mejoran los resultados de la calidad del café, en las variedades Caturra rojo y amarillo, ubicados a altitudes de 420, 570 y $625 \mathrm{~m}$ respectivamente (Cabrera et al., 1991). Otros estudios demostraron diferencias en la composición química de cafeína y lípidos en variedades Borbón y Catuai para altitudes entre 1100 y 1400 m (Guyot et al., 1996). Según Avelino et al., (2005); las plantaciones con pisos altitudinales entre 1020 y $1250 \mathrm{~m}$ presentan mayor acidez, en comparación con el café de altitudes entre 1550 y $1780 \mathrm{~m}$. Bertrand et al., (2006); reporta que a mayor piso altitudinal incrementa el contenido de ácidos clorogénicos y lípidos.

Teniendo en cuenta los elementos anteriormente expuestos, se plantea el presente trabajo con el objetivo de evaluar los parámetros físicos-químicos y sensoriales de la pulpa de café (Coffea arábica), en tres 
variedades: Catuaí, Lempira y Parainema y tres pisos altitudinales $(1275 \mathrm{msnm}, 1461 \mathrm{msnm}$ y 1550 msnm).

\section{MATERIALES Y METODOS}

El método empleado en esta investigación es de enfoque mixto y de orden transversal mediante visitas de campo y análisis de laboratorio. Siguiendo la metodología con ligeras modificaciones de Marcía et al., 2019a.

Las muestras de café variedad Catuaí, Lempira y Parainema, se cosecharon en las fincas de Café Orgánico Márcala, Sociedad Anónima (COMSA), en estado óptimo de maduración en diferentes pisos altitudinales de 1275, 1461 y $1550 \mathrm{msnm}$ respectivamente, a una temperatura de $20 \pm 5^{\circ} \mathrm{C}$. Posteriormente se realizó un prelavado con agua potabilizada, hasta retirar todo el material extraño, luego se dejó reposar en un recipiente plástico adicionando agua hasta cubrirlo completamente durante 30 minutos, logrando que la pulpa se desprendiera del grano y se facilitara el despulpado. Después de 3 h de la cosecha se despulpo mecánicamente, la pulpa que se obtuvo fue depositada en sacos limpios, para protegerla de daños tipo físicos y químicos. La pulpa se secó en secadores solares tipo domo a una temperatura de $40^{\circ} \mathrm{C}$ y una humedad relativa del $60 \%$ durante 10 días. Finalmente, la pulpa de café se molió en un molino eléctrico automático de granos modelo Cuisinart, con tamaño de partícula gruesa que oscila 701 a $900 \mu \mathrm{m}$.

La caracterización físico-química se realizó a partir de $1000 \mathrm{~g}$ de muestra de pulpa de café para cada variedad y piso altitudinal, subdividiéndose en muestras homogenizadas de $100 \mathrm{~g}$ para un mejor manejo experimental, desarrollando los análisis físico-químicos y sensorial por triplicado.

La determinación del potencial de hidrogeno $(\mathrm{pH})$, se realizó por técnica instrumental, empleando un Potenciometro Eutech Instruments con lectura de 0 a $14 \mathrm{de} \mathrm{pH}$, a partir de $100 \mathrm{~g}$ de muestra dentro de un Beacker Labconco de $250 \mathrm{ml}$. El contenido de solidos solubles se determino mediante la tecnica de refractometría empleando un refractometro BOECO con escala de 0 a $90^{\circ} \mathrm{Bx}$, a partir de $1 \mathrm{~g}$ de muestra. Para el analisis sensorial se utilizo $10 \mathrm{~g}$ de café de las distintas variedades y pisos altitudinales en 250 $\mathrm{ml}$ de agua a $93^{\circ} \mathrm{C} \pm 1^{\circ} \mathrm{C}$, finalmente los jueces especializados desarrollaron la prueba de catación a partir de pruebas descriptivas.

Para obtener una mejor interpretación de los hallazgos, se utilizó la metodología con ligeras modificaciones de Marcía et al., (2019b); a partir del uso de análisis estadístico, en relación a las formulaciones de prueba, utilizando el programa IBM, SPSS, versión 25.0 con un 95\% de confianza para la determinación de medias, desviación estándar y estadígrafos con un $\mathrm{p} \leq 0.05$.

\section{RESULTADO Y DISCUSIÓN}

La figura 1 muestra los resultados obtenidos en la determinación de solidos solubles en relación a los pisos altitudinales, demostrando que a mayor piso altitudinal se evidencia mejor calidad química de la pulpa, siendo las variedades Catuaí y Lempira quienes presentan mayor contenido con $15.9^{\circ} \mathrm{Bx}$ y $15.85^{\circ}$ $\mathrm{Bx}$ a una altitud de $1550 \mathrm{msnm}$ respectivamente. Estos resultados ratifican lo expuesto por Pleissner et al., (2016); que indicaron que, a mayor altitud la pulpa de café, contiene más contenido de azucares. 


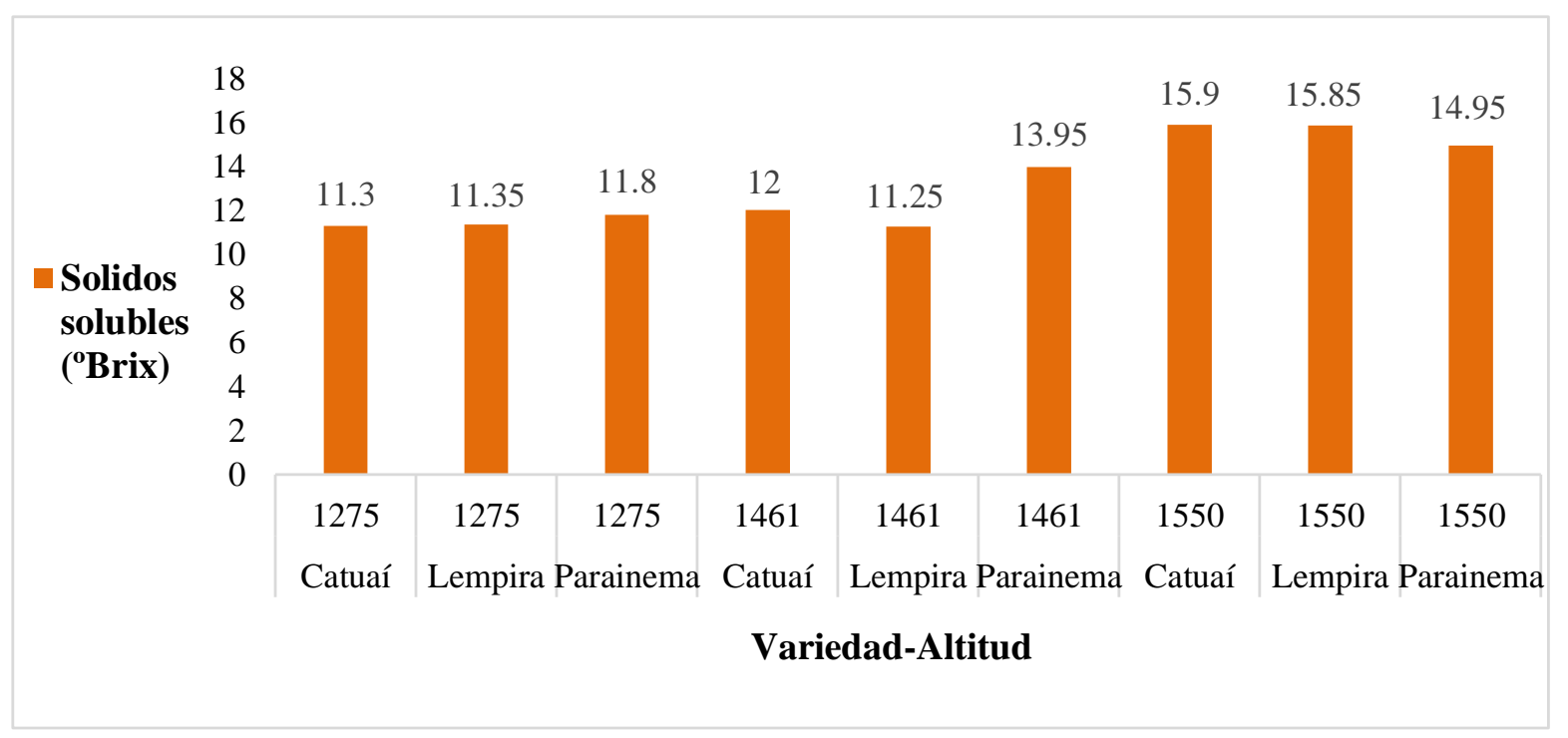

Figura 1. Determinación de solidos solubles de la pulpa de las diferentes variedades de café en distintos pisos altitudinales.

La figura 2 resume los resultados obtenidos del potencial de hidrogeno de la pulpa de las variedades de café en diferentes pisos altitudinales, donde la variedad Parainema evidencia un pH de 5.75 a 1550 $\mathrm{msnm}$, generando diferencia entre el resto de pruebas. Estos resultados discrepan por lo expuesto por Fierro (2018); y Blandón et al., (1999); que reportaron un pH menor de 5 a un piso altitudinal entre 1200 y $1500 \mathrm{msnm}$, para la misma variedad de pulpa de café.

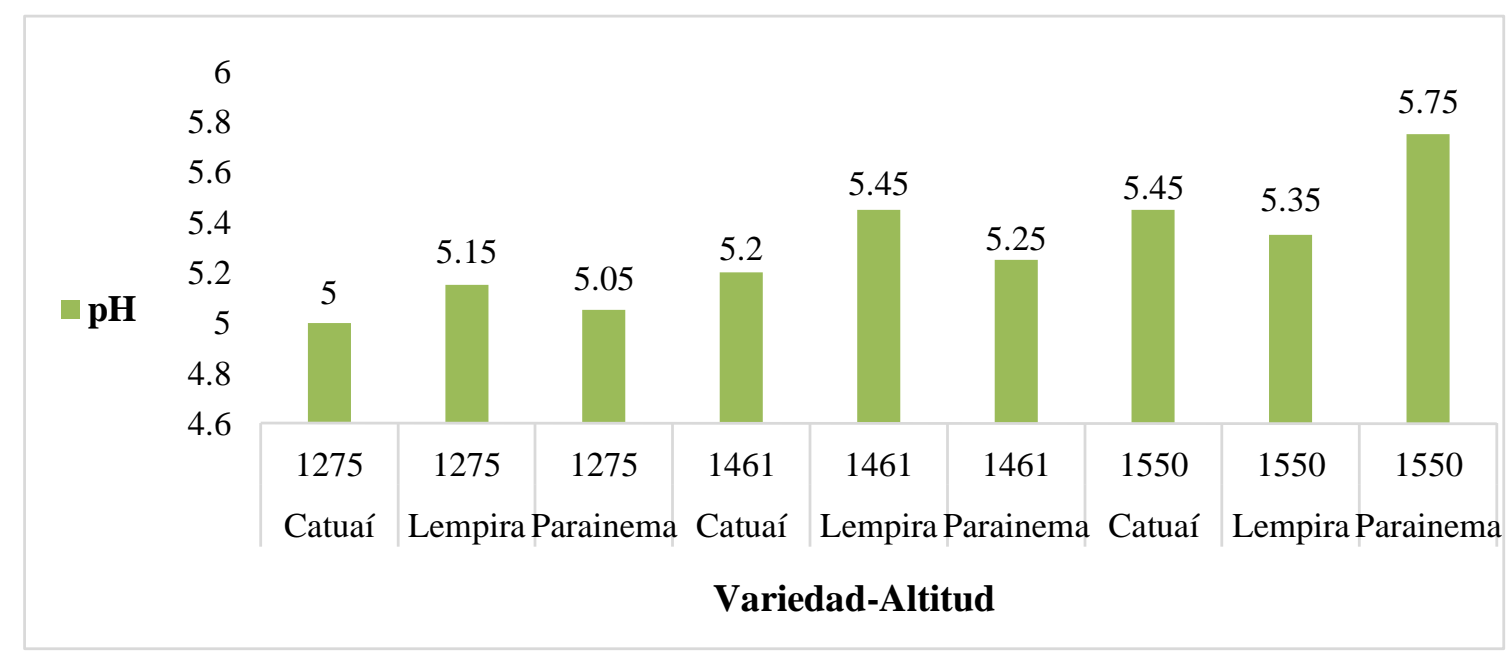

Figura 2. Determinación del pH de la pulpa de las diferentes variedades de café en distintos pisos altitudinales.

La figura 3 muestra los resultados obtenidos de la bebida tipo infusión a partir de evaluación sensorial de perfil en taza para cada una de las variedades y pisos altitudinales, obteniendo mayor aceptación con un 83\% a 1550 msnm la variedad Catuaí, describiéndolo como un producto muy agradable con una tonalidad a varias frutas. 


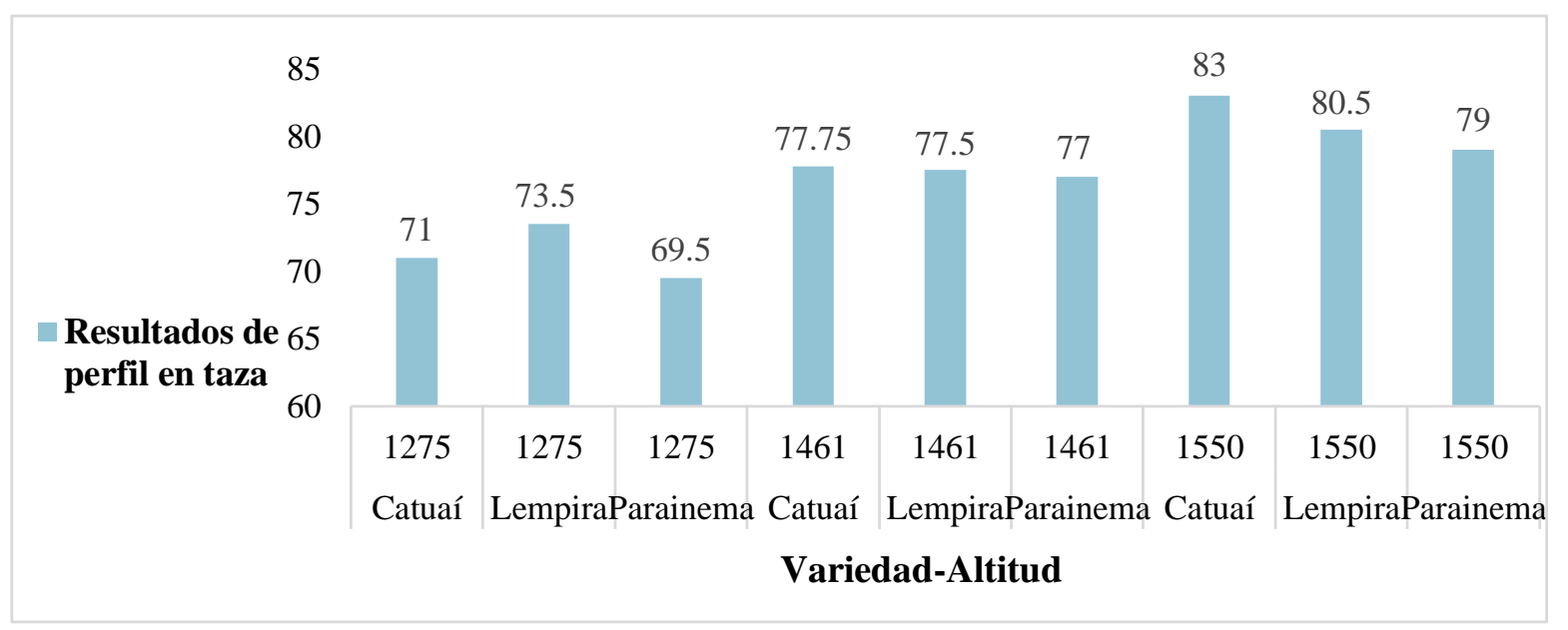

Figura 3. Evaluación sensorial perfil en taza de la bebida tipo infusión.

\section{CONCLUSIONES}

Esta investigación evidencio que el piso altitudinal se relaciona con el contenido de solidos solubles y aceptación sensorial, siendo directamente proporcional.

La variedad Catuaí sobresale en perfil en taza con relación a la variedad Parainema y Lempira, generando atributos sensoriales que promueven un sabor frutal en una bebida tipo infusión a partir de la pulpa.

La industria del café no aprovecha en su totalidad este cultivo, ocasionando mermas en el proceso de manufactura del grano, principalmente por el desaprovechamiento de la pulpa, que podría emplearse en el desarrollo de nuevos productos con características funcionales.

\section{REFERENCIAS}

Avelino, J., Barboza, B., Araya, J.C., Fonseca, C., Davrieux, F., Guyot, B., Cilas, C. (2005). Effects of slope exposure, altitude and yield on coffe quality in two altitude terroirs of Costa Rica. Journal of the science of food and agricultura. 85 (11), 1869-1876

Blandón C. G.; Dávila A. M. T.; Rodríguez V. N. (1999) Caracterización microbiológica y físicoquímica de la pulpa de café sola y con mucílago, en proceso de lombricompostaje. Cenicafé. 50(1):523.

Bertrand, B., Vaast, P., Alpizar, E., Etienne, H., Davrieux, F., Charmetant, P. (2006). Comparison of coffe biochemical compositión and bevarage quality of arabica hybrids involving sundance- etiopian origins with traditional varieties at various elevations in Central America. Tree pysiology. 26(9), 12391248 .

Cabrera, S. C., Acevedo, F. A., Lacerra, E. A., Caballero, B. D., Cedeño, C. R. (1991) Algunos índices físicos del café a diferentes alturas del Escambray, Cuba. Centro agrícola. 18(1), 81-96

Coronel, M., \& Marín, A. (2010). Estudio del café especial ecuatoriano. (Tesis inédita de Nutrición y Dietética). Universidad Central de Ecuador, Quito, Ecuador. 
FAO, Food and Agricultural Organization. (1985). Datos agro climatológicos para américa latina y el caribe.

Fierro, N., Contreras, A., González, O., Rosa, E.S., Morales, V. (2018). Caracterización química y nutrimental de la pulpa de café (coffea arabica.). Agroproductividad. 11(4), 9-13.

Guyot, B.; Gueule, D.; Manez, J.C.; Perriot, J.J.; Giron, J.; Villain, L. (1996) Influence de l'altitude et de l'ombrage sur la qualite des cafés Arabica plantantions. Recherce, developpement 3(4);272-280.

IHCAFÉ, Instituto Hondureño del Café (2017). Exportaciones de café cosecha 2016-2017. Honduras. Disponible en www.ihcafe.hn.

Jaramillo R.A. (2005). Clima andino y el café en Colombia. CENICAFE, 3-8.

Marcía Fuentes, J., Montero Fernández, I., Zumbado, H., Lozano Sánchez, J., Santos Alemán, R., Navarro Alarcon, M., Borrás Linares, I., Saravia Maldonado, S. (2020). Quantification of Bioactive Molecules, Minerals and Bromatological Analysis in Carao (Cassia grandis). Journal of Agricultural Science, 12 (3), 88-94.

Marcía Fuentes, J., Alemán Santos, R., Chavarría Carrión, L., Varela Murillo, I., Alvarado, N., Montero Fernández, I. (2019a). Development of a Drink Type Infusion From Coffee Pulp (Coffea arabica) Lempira Variety of Honduras. Journal of Agricultural Science, 12(1), 209-212.

Marcía Fuentes, J., Chavarría Carrión, L., \& Zumbado, H. (2019b). Análisis del proceso de harina de yuca, sobre las propiedades sensoriales y nutricionales del casabe (Artículo Profesional). Nexo Revista Científica, 32(01), 88-93.

Mozaffarian, D. (2016). Dietary and Policy Priorities for Cardiovascular Disease, Diabetes, and Obesity: A Comprehensive Review. Circulation.133 (2), 187-225.

Pleissner, D., Neu, A. K., Mehlmann, K., Schneider, R., Puerta, G. I., Quintero, J. V. (2016). Fermentative lactic acid production from coffee pulp hydrolysate using Bacillus coagulans at laboratory and pilot scales. Bioresource Technology. 218(10), 167-173

Quintero, G.I.; Gonzales, F.; Correa, A.; Álvarez, I., Ardila, J.; Girón, O.; Ramírez, C.; Baute, J.; Sánchez, P.; Santamaría, M.; Montoya, D. (2016). Diagnóstico de la calidad del café según altitud, suelos y beneficio en varias regiones de Colombia. Cenicafé, 67(2); 15-51.

Serna, O. A.; Torres, L. S.; Martínez, K., Hernández, M. S. (2018). Aprovechamiento de la pulpa de café como alternativa de valorización de subproductos. Rev. Ion. 31(1),37-42.

Ulloa, J. B., Verreth, J. A. J., van Weerd, J. H., \& Huisman, E. A. (2002). Effect of different chemical treatments on nutricional and antinutritional properties of coffee pulp. Animal Feed Science and Technology, 99(1-4), 195-204 
Jhunior Abrahan Marcía Fuentes, Ingeniero Agroindustrial de la Universidad Nacional Autónoma de Honduras.

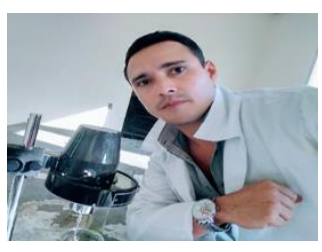
Master en Procesamiento de Alimentos en la Universidad Nacional de Ingeniería de Managua, Nicaragua. Candidato a Doctor en el grado Científico en Alimentos en la Universidad de La Habana, Cuba. Actualmente se desempeña como profesor de la Universidad Nacional de Agricultura de Honduras (UNA), en la Facultad de Ciencias Tecnológicas, en la carrera de Ingeniería en Alimentos. Ganador del Premio Joven Investigador Latinoamericano 2019, del Grupo COIMBRA de Universidades Europeas. Investigador colaborativo de prestigiosas Universidades y Centros de Investigación entre las que destacan Louisiana State University de los Estados Unidos, El Centro de Investigación para el Desarrollo del Alimento Funcional y Nutracéutico, CIDAF y la Universidad de Granada, España. En la actualidad desarrolla investigaciones en compuestos bíoactivos con plantas nativas del continente americano, en las que destacan plantas del Amazona de Brasil y plantas endémicas de Honduras, para su potencial uso como nutracéuticos y bíoconservantes alimenticios.

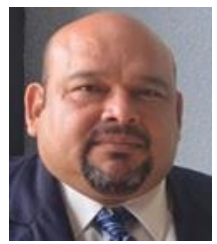

Leonardo Chavarría Carrión, Ingeniero Químico (UNI, 2000). Master en Procesamiento de Alimentos (UNI-2003), Master en Gestión Universitaria (UNI, 2009) coautor del artículo, Investigador en las áreas de: Desarrollo de Nuevos Productos y Seguridad Alimentaria y Nutricional, Profesor titular de la Facultad de Ingeniería Química de la Universidad Nacional de Ingeniería (UNI) Nicaragua, Director del Programa de Vinculación e Innovación Tecnológica (P-VIT). 